\title{
Bone mineral density and bone turnover in spinal osteoarthrosis
}

\author{
N F A Peel, N A Barrington, A Blumsohn, A Colwell, R Hannon, R Eastell
}

\begin{abstract}
Objectives-To determine whether there was a generalised increase in bone mineral density (BMD) in spinal osteoarthrosis (OA), and to determine the mechanism of this possible protection against osteoporosis as assessed by biochemical markers of bone turnover.

Methods-We studied 375 women (ages 50 to 85) from a population based group. Spinal OA was defined from radiographs as the presence of degenerative changes affecting intervertebral or facet joints. BMD of the lumbar spine (LS), femoral neck (FN) and total body (TB) was measured by dual energy $x$ ray absorptiometry (Lunar DPX). Bone turnover rates were estimated from measurement of biochemical markers of bone formation and resorption (urine deoxypyridinoline (Dpyr) and serum bone specific alkaline phosphatase (BAP)).

Results-BMD at each site was greater in the women with spinal OA (mean increase in LS-BMD 7.9\%, 95\% confidence interval (CI) 1.0 to $15 \cdot 1$; TB-BMD 8.4\%, $95 \%$ CI 1.9 to $9 \cdot 7$; FN-BMD $6 \cdot 4 \%, 95 \%$ CI 0.3 to $12 \cdot 6$ ). Twenty four hour urinary excretion of Dpyr, corrected for TB bone mineral content, and serum BAP were $19 \%$ lower in the women with spinal OA $(95 \%$ CI for Dpyr 4.3 to 31.9\%; for BAP 6.3 to $32 \cdot 0 \%$ ). Conclusions-Spinal $\mathrm{OA}$ is associated with a generalised increase in BMD and a decreased rate of bone turnover. This suggests that the protective effect of spinal OA against osteoporosis may be mediated by decreased bone turnover.
\end{abstract}

(Ann Rheum Dis 1995; 54: 867-871)

Patients with osteoarthrosis (OA) may have increased bone mineral density (BMD). ${ }^{12}$ Those with generalised OA have been shown to have increased bone mineral content (BMC) of the radius ${ }^{34}$ and those with OA of the hip have been shown to have increased BMD of the hip estimated from radiological assessments. ${ }^{5}$ Dequeker showed a negative association between radiological scores for $O A$ and osteoporosis in postmenopausal women, ${ }^{6}$ and more recently, Masud et $a l^{7}$ demonstrated increased BMD of the lumbar spine (LS) in women with vertebral osteoporosis who also had spinal OA. These women also had greater
BMD of the femoral neck (FN), although the increase at this site was smaller. Increased $\mathrm{BMD}$ in the presence of OA may, therefore, be greatest in the region affected by OA, but may also affect the skeleton generally.

An increase in measured $\mathrm{BMD}$ in a region affected by OA may result from artefactual error caused by the presence of osteophytes, joint space narrowing and sclerosis within the region of interest. Alternatively, it may reflect a generalised effect of OA on the skeleton, or a combination of the two. If OA has a generalised effect on the skeleton, then study of bone metabolism in patients with OA may be important in understanding the pathophysiology of osteoporosis.

Studies in patients with OA are complicated by the heterogeneous nature of the disease and by the poor correlation between symptoms and radiological signs. ${ }^{8}$ It is therefore necessary to determine the prevalence of OA from radiological surveys. It is also unknown whether all degenerative changes identified on radiographs are pathological, or whether minor changes may represent physiological changes of aging.

There has been little study of the underlying mechanism of the interaction between bone mass and OA. The development of noninvasive biochemical assays for markers of bone resorption and formation enable estimation of bone turnover in large cohorts of subjects. The urinary excretion of pyridinium crosslinks has been shown to be increased in patients with large joint $\mathrm{OA},{ }^{9-12}$ suggesting an increased rate of bone turnover in this condition; however, none of these studies allowed for the fact that there may have been an increased skeletal mass to account for the increase in crosslink excretion. There have been no reports of biochemical markers in subjects with spinal OA.

It is now possible to make accurate and precise measurements of $\mathrm{BMD}$ using dual energy $x$ ray absorptiometry (DXA). This technique enables measurement of the $\mathrm{BMD}$ of the whole skeleton, in addition to measurements at the $\mathrm{LS}$ and FN. It is also possible to make accurate measurements of the BMC of the whole skeleton which can be used to normalise the measurement of the resorption markers to skeletal mass.

The aims of this study were to determine whether the presence of spinal OA was associated with a generalised increase in $\mathrm{BMD}$, and if so, to determine the underlying mechanism at a tissue level. \\ Mepartment of $\mathbf{H}$.tabolism and
Clinical Biochem
}




\section{Subjects and methods \\ SUBJECTS}

We studied 375 women aged 50 to 85 (mean 64) years, randomly selected from three general practice populations in Sheffield. Women were excluded if their GP felt they were too ill to take part (for example because of terminal illness) or if they were unable to give informed consent. Among those invited to participate, the uptake rate was $55 \%$.

\section{SPINAL OSTEOARTHROSIS}

Each subject had anteroposterior and lateral radiographs of the thoracic and lumbar spine. Radiographs were taken with a film to target distance of $100 \mathrm{~cm}$. Thoracic films were centred on vertebra T7 and lumbar films on L2. Spinal OA was defined from the radiographs according to the criteria of Kellgren and Lawrence. ${ }^{13}$ Disc degeneration was defined as the presence of marginal osteophytes, sclerosis of the vertebral plates, or narrowing of the disc space. Facet joint degeneration was defined as the presence of osteophytes or subchondral sclerosis. In the case of both intervertebral and facet joints, changes were graded from 0 to 4 , on the basis of the most severe changes present: $0=$ no changes; $1=$ doubtful; $2=$ minimal; $3=$ moderate; $4=$ severe. Grades 2 to 4 were taken to represent the presence of OA. The scores for the facet joints and the intervertebral joints were added to give a composite score for spinal OA. The radiographs were all reviewed by a single observer, and the within observer variability, assessed by rescoring 50 radiographs, demonstrated a kappa score of $0 \cdot 76$.

\section{BONE DENSITOMETRY}

BMD of the LS, TB, and FN were measured by DXA (Lunar DPX, Lunar Corp, Madison, WI). The reproducibility of these measurements, assessed from duplicate measurements in 20 women from the population based group, were $0.8 \%$ for $\mathrm{LS}-\mathrm{BMD}, 1 \cdot 0 \%$ for TB-BMD, and $2.9 \%$ for FN-BMD. In the case of subjects with a vertebral deformity within the region of analysis for the LS-BMD measurement (L2-L4), the deformed vertebra was excluded from the analysis.

\section{VERTEBRAL DEFORMITIES}

Vertebral deformities were defined from the spinal radiographs using morphometric criteria. ${ }^{14}$ In this method the anterior (ha), posterior

Table 1 Characteristics of women with and without spinal osteoarthrosis (OA) from a population based group

\begin{tabular}{|c|c|c|c|c|}
\hline & $\begin{array}{l}\text { Spinal OA } \\
(n=340)\end{array}$ & $\begin{array}{l}\text { No spinal } O A \\
(n=35)\end{array}$ & $p$ & $95 \%$ CI of diff. \\
\hline $\begin{array}{l}\text { Age (yr) } \\
\text { Age at menopause (yr) }+ \\
\text { Height }(\mathrm{m}) \\
\text { Weight }(\mathbf{k g})\end{array}$ & $\begin{array}{l}64 \cdot 7(9 \cdot 05) \\
47 \cdot 7(5 \cdot 2) \\
1 \cdot 58(6 \cdot 5) \\
66 \cdot 3(12 \cdot 5)\end{array}$ & $\begin{array}{l}61 \cdot 8(8 \cdot 16) \\
46 \cdot 8(4 \cdot 5) \\
1 \cdot 57(5 \cdot 6) \\
62 \cdot 8(9 \cdot 9)\end{array}$ & $\begin{array}{l}0 \cdot 09 \\
0 \cdot 4 \\
0 \cdot 2 \\
0 \cdot 1\end{array}$ & $\begin{array}{l}-6.2 \text { to } 0.5 \\
-1.2 \text { to } 3.1 \\
-0.4 \text { to } 0.08 \\
-8.1 \text { to } 1.1\end{array}$ \\
\hline
\end{tabular}

Values are mean (SD).

†For 281 women with spinal $O A$, and 24 women without spinal OA; not determined in the remaining women because of factors such as surgical hysterectomy with conservation of ovaries before the natural menopause. CI = Confidence interval. (hp), and mid height (hm) of each vertebra from T4 to L5 were measured on the lateral radiographs and deformities defined for each vertebra as:

$\%$ wedge $=(\mathrm{hp}-\mathrm{ha}) / \mathrm{hp} \times 100$

$\%$ biconcavity $=(\mathrm{hp}-\mathrm{hm}) / \mathrm{hp} \times 100$

$\%$ compression $=\left(h^{\prime}-h p\right) / h^{\prime} \times 100$

where hp' is the posterior height of the vertebra below or the vertebra above. The deformities for each vertebra were compared with published normative data from women established by Eastell $e t$ al $^{14}$ and a vertebra was considered to be fractured if it had a deformity more than 3 SD less than the mean for that vertebra.

BIOCHEMICAL MARKERS OF BONE TURNOVER Fasting blood was collected from each subject between 09:00 and 09:30. Each subject also made a 24 hour urine collection. Samples were stored at $-70^{\circ} \mathrm{C}$ before measurement. Pyridinoline (Pyr) and deoxypyridinoline (Dpyr) were measured in $\mathbf{2 4}$ hour urine collections by high performance liquid chromatography and fluorescence detection. ${ }^{15}$ Excretion of crosslinks was normalised to TB-BMC, and to creatinine excretion (Cr). Serum activity of bone specific isoenzyme of alkaline phosphatase (BAP) was measured using a wheatgerm lectin precipitation assay. ${ }^{16}$

\section{STATISTICAL ANALYSIS}

Comparisons between groups were made using unpaired $t$ tests after logarithmic transformation to approximate a normal distribution where appropriate. $\mathrm{Z}$ scores, expressed as standard deviations from expected, were calculated by normalising BMD for age and weight in a multiple linear regression model. The relationship between variables was assessed using Pearson correlation coefficients for parametric variables, with logarithmic transformation where appropriate, and Spearman rank correlation for variables not normally distributed. The odds ratio and $95 \%$ confidence interval (CI) for vertebral deformity were calculated using the method of Gardner and Altman. ${ }^{17}$ Analyses were performed using Statgraphics Statistical Graphics System (Statistical Graphics Corporation, Rockville, MD).

\section{Results}

Table 1 compares the 340 women with and 35 women without spinal OA in terms of age, age at menopause, height and weight.

BONE MINERAL DENSITY

Table 2 shows the BMD of the women with and without spinal OA. Both absolute values of $B M D$ and $Z$ scores were greater in the women with OA. Figure 1 shows the comparison of $Z$ scores at each skeletal site.

\section{PREVALENCE OF VERTEBRAL DEFORMITIES}

Twenty two women with spinal OA (6.5\%) had vertebral deformities, compared with four 
Table 2 Comparison of bone mineral density (BMD) at three skeletal sites in 375 women from a population based group according to presence of spinal osteoarthrosis (OA)

\begin{tabular}{llllll}
\hline & Spinal OA & No spinal OA & Difference & $p$ & $95 \%$ CI of diff. \\
\hline LS-BMD $\left(g / \mathrm{cm}^{2}\right)$ & $1.073(0.19)$ & $0.994(0.18)$ & $7.9 \%$ & 0.02 & 1.0 to $15.1 \%$ \\
TB-BMD $\left(g / \mathrm{cm}^{2}\right)$ & $1.086(0.11)$ & $1.027(0.11)$ & $8.4 \%$ & 0.005 & 1.9 to $9.7 \%$ \\
FN-BMD (g/cm $)$ & $0.846(0.13)$ & $0.795(0.13)$ & $6.4 \%$ & 0.04 & 0.3 to $12.6 \%$ \\
LS Z score (SD units) & $0.03(1.00)$ & $-0.41(1.00)$ & 0.44 & 0.02 & 0.07 to 0.82 \\
TB Z score (SD units) & $0.12(1.04)$ & $-0.54(0.99)$ & 0.66 & 0.004 & 0.30 to 1.02 \\
FN Z score (SD units) & $0.27(1.00)$ & $-0.21(0.95)$ & 0.48 & 0.009 & 0.12 to 0.85 \\
\hline
\end{tabular}

Values are mean (SD). Difference between groups shown as increase relative to women with no OA. $\mathrm{LS}=$ Lumbar spine; TB = total body; $\mathrm{FN}=$ femoral neck.
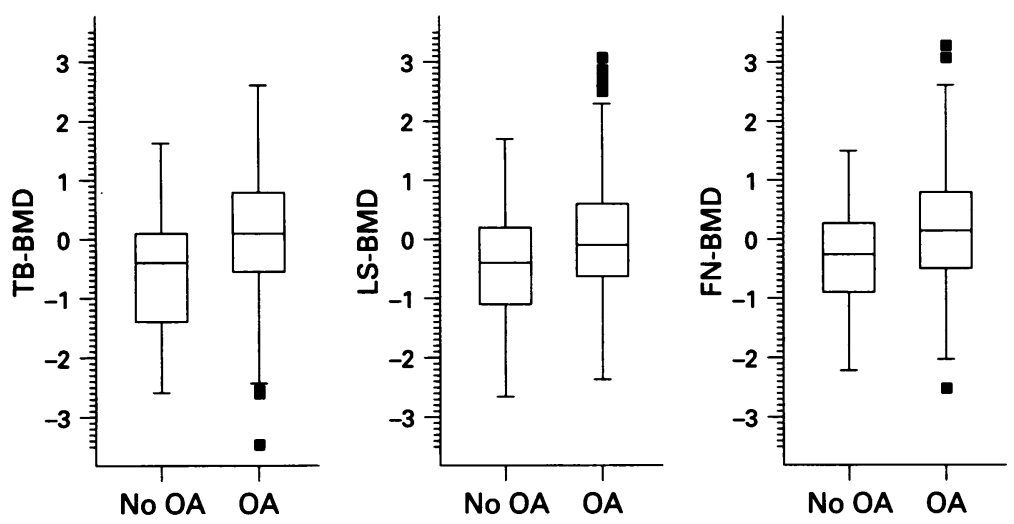

Figure 1 Comparison of bone mineral density (BMD) $Z$ scores at three skeletal sites in women with and without spinal osteoarthrosis $(O A)$ from a population based group. Values are median (horizontal line), interquartile range (box), and range (whiskers). Outliers, shown as discrete data points, are those values $>1.5 \times$ interquartile range above or below interquartile range. Difference between women with and without $O A$ significant at each site: total body (TB) $p=0.0004$; lumbar spine (LS) $p=0.02$; femoral neck (FN) $p=0.009$.

$(11.4 \%)$ of the women without spinal OA. The odds ratio for vertebral deformity in women with spinal OA was decreased $(0 \cdot 536)$, but this was not statistically significant $(95 \% \mathrm{CI}$ $0 \cdot 2$ to $1 \cdot 7)$.

Table 3 Comparison of biochemical markers of bone resorption and bone formation in women with and without spinal osteoarthrosis $(O A)$ from a population based group

\begin{tabular}{llccc}
\hline & Spinal OA & No spinal OA & $\begin{array}{l}\text { Decrease in } \\
\text { spinal } O A \\
\text { (\%) }\end{array}$ & $\begin{array}{l}\text { 95\% CI of diff. } \\
\text { (\%) }\end{array}$ \\
\hline Dpyr/TB-BMC (nmol/day/g) & $0.051(0.02)$ & $0.063(0.02)$ & 19 & 4.3 to 31.9 \\
Dpyr/Cr/nmol/mmol) & $0.011(0.008)$ & $0.013(0.02)$ & 18 & -54.3 to 80.9 \\
Pyr/TB-BMC (nmolday/g) & $0.165(0.07)$ & $0.193(0.06)$ & 14 & 1.5 to 31.5 \\
Pyr/Cr (nmol/mmol) & $0.037(0.08)$ & $0.039(0.03)$ & 5 & -63 to 83 \\
BAP (IU//) & $37.9(15.6)$ & $46.8(20.9)$ & 19 & 6.3 to 32.0 \\
\hline
\end{tabular}

TB-BMC = Total body bone mineral content; $\mathrm{Cr}=$ creatinine; Dpyr = deoxypyridinoline; $\mathrm{Pyr}=$ pyridinoline; $\mathrm{BAP}=$ bone specific alkaline phosphatase.
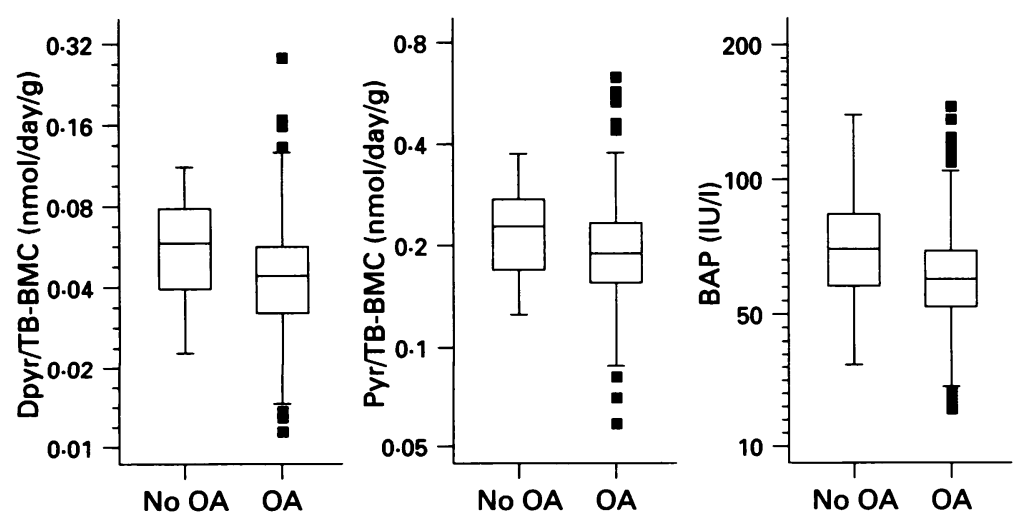

Figure 2 Comparison of biochemical markers of bone turnover in women with and without Figure 2 Comparison of biochemical markers of bone turnover in women with and without
spinal osteoarthrosis (OA) from a population based group. Values are median, interquartile range, and range as in figure 1. Difference between the women with and without spinal $O A$ significant for markers of both resorption (deoxypyridinoline (DPyr) TB-BMC, $p=0.01$; pyridinoline (Pyr) TB-BMC, $p=0.05$ ) and formation (bone specific alkaline phosphatase $(B A P) p=0.003)$. TB-BMC = Total body bone mineral content.
BONE TURNOVER

Table 3 shows the difference in biochemical markers of bone turnover in the women with and without spinal OA. Results for the urinary excretion of pyridinium crosslinks are shown both after normalisation for creatinine and normalisation for TB-BMC. Figure 2 illustrates the difference in bone turnover markers.

Table 4 shows the relationship between the severity of spinal OA, BMD, and biochemical markers of bone turnover, showing that the severity of OA changes was associated with BMD and negatively associated with bone turnover. BMD, normalised for age and weight, was highly negatively correlated with bone turnover. Figure 3 shows the relationship between bone turnover and TB Z score. Spinal OA score correlated (Spearman rank correlation) with age $(r=0.24, \mathrm{p}<0.001)$, years postmenopause $\quad(r=0.23, \mathrm{p}=0.001)$, and weight $(r=0.15, p=0.006)$. The correlation of spinal $\mathrm{OA}$ score with absolute $B M D$ values was weaker than that with $B M D$ $\mathrm{Z}$ scores (TB-BMD $r=0.16, \mathrm{p}=0.004$; LS-BMD $r=0.28, \mathrm{p}<0.0001 ;$ FN-BMD $r=0.08, \mathrm{p}=0.15$ ).

\section{Discussion}

We found that radiological evidence of spinal OA was very common in this population based group of women. The age of the women with spinal OA was not greater than that of women without spinal OA, but we did find that the severity of spinal OA changes increased with age. Similarly, though there was no difference between the weight of the women with and without spinal $O A$, increasing body weight was associated with more severe OA. This suggested that perhaps women with no radiological evidence of degenerative spinal disease should be considered as a discrete group. Accordingly, we compared $\mathrm{BMD}$ and bone turnover in the women with and without spinal OA.

We found that BMD was increased in the women with spinal OA, not only at the lumbar spine, but also at the femoral neck and total body. The increase in BMD at each site remained after correction for age and weight. Increased LS-BMD in the presence of spinal OA may result from artefactual increases due to the presence of osteophytes, intervertebral joint space narrowing, and facet joint sclerosis within the area of analysis. ${ }^{18} 19$ Although an association has been shown between spinal OA and $\mathrm{OA}$ of the hip, ${ }^{20}$ the area selected for BMD analysis at the hip (the femoral neck) is not affected by joint space narrowing, osteophytes or periarticular sclerosis. This suggests that there is a real increase in FN-BMD in women with spinal OA. We also found an increase in TB-BMD. This could reflect a generalised increase in $B M D$, or may reflect $O A$ at sites other than the spine in association with spinal OA. We do not have radiographs of other skeletal sites in these women and the association between radiological osteoarthrosis and symptoms of pain and stiffness is poor. We have therefore been unable to ascertain 
Table 4 Correlation matrix showing relationship between bone mineral density (BMD), bone turnover and severity of spinal osteoarthrosis $(O A)$ in 375 women from a population based group

\begin{tabular}{lllll}
\hline & Spinal OA score & $\begin{array}{l}\text { Dpyn/TB-BMC, } \\
(\text { nmolday/g) }\end{array}$ & $\begin{array}{l}\text { Pyr/TB-BMC } \\
(\text { nmolday/g) }\end{array}$ & BAP (IUn) \\
\hline Spinal OA score & - & $-0 \cdot 15^{\star \star}$ & $-0 \cdot 10$ & $0 \cdot 04$ \\
TB-BMD Z score & $0 \cdot 26^{\star \star \star}$ & $-0 \cdot 38^{\star \star \star}$ & $-0 \cdot 32^{\star \star \star}$ & $-0 \cdot 32^{\star \star \star}$ \\
LS-BMD Z score & $0 \cdot 16^{\star \star}$ & $-0 \cdot 30^{\star \star \star}$ & $-0 \cdot 22^{\star \star \star}$ & $-0 \cdot 18^{\star \star}$ \\
FN-BMD Z score & $0 \cdot 31^{\star \star}$ & $-0 \cdot 26^{\star \star \star}$ & $-0 \cdot 20^{\star \star \star}$ & $-0 \cdot 19^{\star \star}$ \\
\hline
\end{tabular}

Values are correlation coefficients. $\mathbf{B M C}=$ Bone mineral content; $\mathbf{B M D}=$ bone mineral density; TB = total body; LS = lumbar spine; FN = femoral neck; Dpyr = deoxypyridinoline; Pyr pyridinoline; $\mathrm{BAP}=$ bone specific alkaline phosphatase. ${ }^{\star} \mathrm{p}<0.05,{ }^{\star \star} \mathrm{p}<0.01,{ }^{\star \star \star} \mathrm{p}<0.001$.

whether the increase in TB-BMD was associated with generalised $\mathrm{OA}$.

LS-BMD is the major determinant of vertebral facture risk, accounting for between 75 and $85 \%$ of the variability in bone strength. ${ }^{21}$ It has been shown that the risk of vertebral facture increases two- to threefold for each SD decrease in BMD below the mean. ${ }^{22}$ We would therefore expect spinal OA to have a protective effect against vertebral facture. We found that the odds ratio for vertebral deformity in the women with spinal OA was decreased, but that this did not reach statistical significance. This may reflect the low prevalence of vertebral deformity overall in this cohort, and the small number of women with absence of spinal OA.

Measurement of the biochemical markers of bone turnover enabled us to examine the mechanism of the increased $B M D$ in the women with spinal OA. We found a decrease in both resorption and formation markers in the women with spinal OA. This suggests that these women had a lower rate of bone turnover than the women without spinal OA. The urinary excretion of the bone resorption markers, Pyr and Dpyr, is commonly normalised to creatinine excretion as an approximation of body mass, and to allow for incomplete urine collection. However, in this study we were able to normalise crosslink excretion to TB-BMC, which accurately reflects skeletal mass. Whilst the crosslink excretion, corrected for creatinine, was not different between the women with and without spinal OA, there was a significant difference when crosslink excretion was expressed in relation to unit bone mass and the effect of the increase in bone mass was accounted for. This may be one reason why these results differ from previous studies that
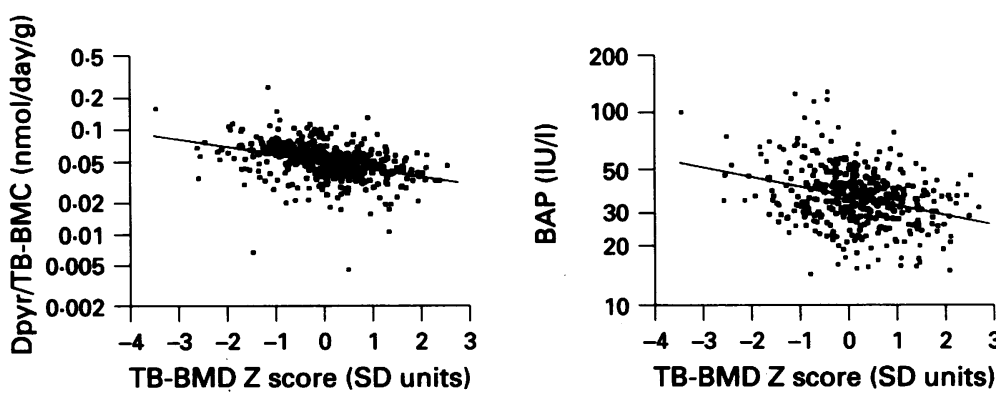

Figure 3 Relationship of bone resorption marker deoxypyridinoline (Dpyr) (left panel) and bone formation marker bone specific alkaline phosphatase (BAP) (right panel) with total body bone mineral density (TB-BMD) normalised for age and weight in 375 woman aged 50 to 85 years. Dpyr: $\mathrm{r}=-0.38, p<0.0001 ; B A P: \mathrm{r}=-0.32, p<0.0001$. have shown an increase in crosslink excretion in patients with OA. ${ }^{9-12}$ These previous studies also examined patients with predominantly large joint $\mathrm{OA}$, in which individual joints may have a greater influence on crosslink excretion as a result of the greater mass of osteophytes.

We also showed a strong correlation between age and weight adjusted BMD and bone turnover in the whole cohort of subjects. This is consistent with the concept that inter individual differences in BMD are partly accounted for by differing rates of bone turnover.

We conclude that radiological spinal $O A$ is associated with a generalised increase in BMD and a decreased rate of bone turnover. These results are consistent with the hypothesis that spinal OA has a protective effect against bone loss, mediated by a lower rate of bone turnover. Alternatively, they may suggest the existence of a small subgroup of women with increased bone turnover, who are susceptible to the development of osteoporosis, and who do not develop physiological degenerative changes of the skeleton with aging.

This project was funded by a project grant from the Arthritis and Rheumatism Council (R44). NFAP was supported by the ARC as a clinical research fellow. We would like to thank $D$ Greenfield for recruiting patients, and A Johnson, A Milne, and $\mathrm{S}$ Bowles for BMD measurements.

1 Knight S M, Ring E F J, Bhalla A K. Bone mineral density and osteoarthritis. Ann Rheum Dis 1992; 51: 1025-6.

2 Hordon L D, Wright V, Smith M A. Bone mass in osteoarthritis. Ann Rheum Dis 1992; 51: 823-5.

3 Cooper C, Poll V, McLaren M, Daunt S O N, Cawley M I D. Alterations in appendicular skeletal mass in patients with rheumatoid, psoriatic, and osteoarthropathy. Ann Rheum Dis 1988; 47: 481-4.

4 Price $T$, Hesp $R$, Mitchell $R$. Bone density in generalized osteoarthritis. I Rheumatol 1987; 14: 560-2.

5 Cooper C, Cook P L, Osmond C, Fisher L, Cawley M I D. Osteoarthritis of the hip and osteoporosis of the proximal femur. Ann Rheum Dis 1991; 50: 540-2.

6 Dequeker J. The relationship between osteoporosis and osteoarthritis. Clin Rheum Dis 1985; 11: 271-96.

7 Masud T, Langley S, Wiltshire P, Doyle D V, Spector T D. Effect of spinal osteophytosis on bone mineral density measurements in vertebral osteoporosis. $B M F$ 1993; 307: 172-3.

8 Lawrence J S, Bremner J M, Bier F. Osteoarthrosis. Prevalence in the population and relationship between symptoms and x-ray changes. Ann Rheum Dis 1966; 25: $1-24$.

9 Seibel M J, Duncan A, Robins S P. Urinary hydroxypyridinium crosslinks provide indices of cartilage and pyridinium crosslinks provide indices of cartilage and 16: 964-70.

10 Thompson $P$ W, Spector T D, James I T, Henderson E, Hart D J. Urinary collagen crosslinks reflect the radiographic severity of knee osteoarthritis. $\mathrm{Br} \mathcal{F}$ Rheumatol 1992; 31: 759-61

11 Macdonald A G, McHenry P, Robins S P, Reid D M Relationship of urinary pyridinium crosslinks to disease extent and activity in osteoarthritis. Br $\mathcal{F}$ Rheumatol 1994; 33: 16-9.

12 Astbury C, Bird H A, Mclaren A M, Robins S P. Urinary excretion of pyridinium crosslinks of collagen correlated with joint damage in arthritis. $B r \mathcal{F}$ Rheumatol 1994; 33: with join.

13 Kellgren J H, Lawrence J S. Epidemiology of chronic rheumatism, vol 2. Atlas of standard radiographs of arthritis. Oxford: Blackwell, 1963.

14 Eastell $R$, Cedel S $L$, Wahner $H$ W, Riggs $B$ L Melton L J, III. Classification of vertebral fractures. Melton L J, III. Classification of

15 Colwell A, Russell R G G, Eastell R. Factors affecting the assay of urinary 3-hydroxypyridinium crosslinks of collagen as markers of bone resorption. Eur $\mathcal{f}$ Clin Inves 1993; 23: 341-9.

16 Rosalki S B, Foo A Y. Two new methods for separating and quantifying bone and liver alkaline phosphatase isoenzymes in plasma. Clin Chem 1984; 30: 1182-6.

17 Gardner M J, Altman D G. Calculating confidence intervals for proportions and their differences. Belfast: The Universities Press, 1989; 28-33.

18 Orwoll E S, Oviatt S K, Mann T. The impact of osteophytic and vascular calcifications on vertebral mineral density measurements in men. $\mathcal{f}$ Clin Endocrinol Metab 1990; 70: 1202-7. 
19 Reid I R, Evans M C, Ames R, Wattie D J. The influence of osteophytes and aortic calcification on spinal minera density in postmenopausal women. $f$ Clin Endocrinol Metab 1991; 72: 1372-4.

20 Belmonte-Serrano Michel B E, Fries J F. The relationship between spinal Michel B E, Fries J F. The relationship between spinal
and peripheral osteoarthritis and bone density measurements. $\mathcal{f}$ Rheumatol 1993; 20: 1005-13.
21 Mazess R B. On aging bone loss. Clin Orthop 1982; 165:

22 Ross P D, Davis J W, Epstein R S, Wasnich R D. Preexisting fractures and bone mass predict vertebral fracture incidence in women. Ann Intern Med 1991; 114: incidence 